Extrême-Orient Extrême-Occident

\section{Extrême-Orient Extrême-Occident}

$32 \mid 2010$

Faux et falsification en Chine, au Japon et au Viêt

Nam

\title{
Du bon usage du faux
}

About the Proper Use of Fakes

\section{Laurent Feller}

\section{(2) OpenEdition \\ Journals}

Édition électronique

URL : http://journals.openedition.org/extremeorient/120

DOI : 10.4000/extremeorient. 120

ISSN : 2108-7105

Éditeur

Presses universitaires de Vincennes

\section{Édition imprimée}

Date de publication : 1 octobre 2010

Pagination : 221-232

ISBN : 978-2-84292-263-4

ISSN : 0754-5010

Référence électronique

Laurent Feller, " Du bon usage du faux », Extrême-Orient Extrême-Occident [En ligne], 32 | 2010, mis en ligne le 01 octobre 2013, consulté le 01 mai 2019. URL : http://journals.openedition.org/ extremeorient/120; DOI : 10.4000/extremeorient.120

Ce document a été généré automatiquement le 1 mai 2019.

(c) PUV 


\title{
Du bon usage du faux
}

\author{
About the Proper Use of Fakes
}

\section{Laurent Feller}

1 Le thème du faux est susceptible de variations presque infinies, où que l'on se situe dans l'espace ou dans le temps, et contraint à poser des problèmes toujours complexes mais qui renvoient au cœur de la réflexion et de l'action humaine. Ce numéro, avec les exemples très divers qu'il mobilise, en est la parfaite illustration.

2 Allant d'une réflexion sur le mensonge d'État que constituent les textes d'abdication d'empereurs déposés à la falsification de données archéologiques, en passant par la modification des généalogies dans le Japon ancien et les contrefaçons de marchandises en Chine et au Japon, les auteurs nous permettent de parcourir une gamme typologique ample. Si elle présente des spécificités propres à l'Asie, elle possède une dimension beaucoup plus vaste, parce que, d'une manière ou d'une autre, les nombreuses questions soulevées ici se retrouvent dans beaucoup d'autres contextes géographiques, historiques et historiographiques

3 Parler de faux et de falsifications contraint en effet à aborder des points tout à fait fondamentaux concernant aussi bien l'économie et la vie sociale que, oserais-je dire, la vie morale : le faux pose autant de problèmes éthiques que de problèmes pratiques.

4 Si l'on veut bien considérer que la finalité de l'enquête historique est de produire un discours de vérité, c'est-à-dire de s'approcher au plus près de la réalité des choses du passé, la question du faux devient immédiatement primordiale et lancinante. Le statut scientifique de l'histoire tient en effet précisément à sa capacité à reconstruire et à représenter, c'est-à-dire à ramener à la vue de tous, une réalité qui n'est plus effectivement présente. Elle est cependant là parmi nous à l'état de traces, parce que les hommes du passé ont laissé des empreintes que les historiens transforment en sources par leur travail de critique et d'analyse. Ces empreintes sont multiformes: nous pensons spontanément aux écrits, mais ils sont bien évidemment loin d'être les seuls à être concernés. Les sociétés sans écriture ont aussi une histoire : elle se trouve à l'état de données brutes enfouies dans le sol où elles constituent des archives d'un type particulier dont l'interprétation doit être faite avant que l'on puisse s'en servir comme d'une source. 
Les documents écrits eux-mêmes, présentant une typologie presque infinie, ne disent pas tous la même chose, n'ayant pas été produits, pour la plupart, afin de répondre aux nécessités de la construction d'un récit historique. Il y a des exceptions, et le dossier chinois en propose une magnifique illustration. La production de textes, quelle que soit leur nature, ne transforme pas immédiatement ceux-ci en sources historiques. Comme les objets enfouis dans le sol, ils doivent d'abord être interprétés avant de pouvoir être utilisés au service du discours historique. Or, les hasards de la transmission des textes ou des objets, les contingences liées à la sédimentation des documents d'archives, ainsi que les conditions même de la production du matériau qui sert de matière première à l'historien, compliquent considérablement sa tâche. À tous ces niveaux en effet, il est nécessaire de s'interroger sur la pertinence de ce que nous avons reçu, sur la nature, la fiabilité et la véracité des informations qui sont transmises, quel que soit le support sur lequel elles se trouvent. Le document n'est jamais un donné. Lui-même élaboré à un moment particulier et dans un lieu donné, il n'a pas en lui-même d'autorité et doit être validé par le travail critique de l'historien qui l'emploie. Il en va de même d'un objet archéologique qui, tiré de son contexte, n'a aucun statut scientifique.

5 S'interroger sur le faux revient ainsi en définitive à réfléchir sur les conditions de possibilité d'un discours historique qui cherche à dire non pas le vraisemblable ou le possible, mais le réel et le vrai. Cette exigence primordiale bute en permanence sur la réalité de la documentation, rétive à se laisser saisir et commenter, à se laisser précisément transformer en source de savoir.

\section{Les falsifications de sources}

6 Les sources peuvent être manipulées de toute sorte de façons. Un document peut être original et authentique et, en même temps, n'être pas sincère dans son contenu. Il peut également être parfaitement faux mais contenir des informations véridiques et fiables : même en histoire, il existe un "mentir vrai » qui oblige à la plus grande cautèle. Pour un médiéviste de profession, ces questions sont essentielles, parce qu'elles se trouvent aux origines mêmes de sa discipline et au cœur de son travail: on se souvient de ce que la méthode historique est née, au XVIII ${ }^{\mathrm{e}}$ siècle, de la nécessité de savoir reconnaître les documents falsifiés des documents sincères et, en même temps, de la question de la créance à accorder aux récits hagiographiques. La volonté de démêler le vrai du faux et le soupçon que les choses ne sont jamais ce qu'elles paraissent être se trouvent ainsi au commencement de notre savoir. Le dossier présenté ici propose quelques beaux exemples de falsifications volontaires de la documentation afin de produire un " effet d'histoire ».

7 L'affaire de la falsification des outils paléolithiques que présente Arnaud Nanta est, à cet égard, tout à fait extraordinaire, à défaut (heureusement) d'être caractéristique ou emblématique. Placer des objets sur un chantier de fouilles la nuit, à l'intérieur des couches que l'on s'apprête à fouiller le lendemain, est une vilaine affaire. C'est ce qu'a fait pour des motifs complexes un archéologue japonais soucieux de donner une place particulière au paléolithique. Que cela ait été possible établit d'abord que les conditions mêmes de la fouille n'étaient pas très scrupuleuses, les fouilleurs n'étant pas amenés à s'interroger sur le point de savoir si la strate découverte était ou non en place. C'est d'autre part un pur non-sens scientifique qui peut alors proposer un paradoxe mineur mais intéressant: les objets trouvés peuvent être en eux-mêmes parfaitement authentiques, mais le fait qu'ils n'aient pas été trouvés à l'intérieur d'une couche dont le 
sceau est brisé par l'archéologue avant la procédure de découverte ou d'invention de l'objet qu'est la fouille, les transforme en faux. Autrement dit, leur statut n'est pas meilleur que s'ils avaient été fabriqués pour l'occasion par un artisan, parce que c'est ici le geste même de l'archéologue qui authentifie l'objet. Il est alors comme le notaire dont l'acte garantit la réalité du négoce en train de s'opérer. En tout cas, l'objet découvert perd toute signification scientifique et ne peut plus être considéré comme étant une source valide.

enjeux de telles falsifications sont repérés depuis longtemps : vouloir faire parler les archives du sol n'est pas toujours dépourvu d'arrière-pensées idéologiques ou politiques. Ces faits sont bien connus: la pression du milieu ambiant sur les archéologues est évidemment très considérable, dès lors qu'une découverte peut contribuer à conforter ou à remettre en cause une trame narrative tenue pour acquise et ayant la force d'une vérité d'évidence ou le pouvoir évocateur d'un mythe d'origine.

9 L'attitude de l'archéologue Fujimura Shin'ichi relève évidemment d'une grande malhonnêteté. Elle s'éclaire et se comprend si l'on accepte l'idée de la présence d'enjeux idéologiques forts à l'intérieur de la recherche scientifique. Dans cette optique, les faits ne sont que des arguments dans un débat qui est avant tout politique et surtout passionnel, puisqu'il touche au discours sur les origines de la nation ou du peuple. Dans ce cas, les manipulations des faussaires ne sont pas aisées à découvrir : il faut en fait un flagrant délit, la falsification des rapports de fouilles permettant de masquer les incohérences et le soupçon d'un tel manque de probité scientifique étant extrêmement difficile à formuler dans un milieu académique. D'autre part, les enjeux sont eux-mêmes fort complexes et rarement tout à fait déshonorants.

10 Faire croire à la présence de matériel lithique dans des couches qui ne devraient pas en contenir, ou qui devraient en contenir d'une tout autre nature, est alors un moyen d'imposer une vision de l'histoire en rupture avec le savoir commun de la fin du $\mathrm{xx}^{\mathrm{e}}$ siècle et, accessoirement, d'assurer ou de conforter une position sociale liée à une forme de pouvoir intellectuel.

11 L'archéologue touche à la matière. Sa preuve, ou sa source, est une chose concrète que l'on peut voir et toucher. D'autres falsifications de sources peuvent se trouver qui, jouant sur le récit de faits établis, peuvent plonger le lecteur dans des abîmes de perplexité, comme dans le cas des textes d'abdication sous les Six Dynasties analysés par François Martin. Les actes par lesquels les souverains abandonnent le pouvoir sont présentés comme étant effectués de la pleine volonté du souverain qui affirme juger qu'il y a mieux placé que lui, à certains moments, pour exercer le mandat du ciel. Les actes d'abdication proposent ainsi un dispositif narratif qui justifie la position du successeur et fait du souverain en train d'abdiquer un homme agissant pour le mieux et s'efforçant avant tout de maintenir l'harmonie dans l'Empire. Le document ainsi produit, parfaitement authentique, est aussi parfaitement mensonger, parce qu'il raconte une histoire qui n'a pas eu lieu ou bien s'est déroulée autrement, le souverain abdiquant étant en réalité chassé du pouvoir et, bien souvent, promis à une mort rapide. L'histoire officielle, quelque fausse qu'elle soit, est cependant aussi nécessaire au maintien de l'ordre et de la cohésion politique et sociale: elle produit une impression d'ordre et de stabilité nécessaire au fonctionnement convenable des institutions. Proposant ou plutôt imposant une fiction acceptable par tous, l'acte d'abdication permet d'établir un récit à la véracité duquel les acteurs peuvent feindre de croire sans perdre la face ni leur place dans les institutions, tout en donnant l'impression que les règles et les rites ont à tout moment été 
respectés. La forgerie d'un document officiel qui donne une version solennelle de faits avérés mais le fait de façon totalement biaisée est un pur acte de propagande qui protège l'usurpateur et condamne le ou les vaincus. La falsification atteint ici le cœur même des rapports humains en ceci qu'elle crée une "communauté textuelle » reposant sur des mensonges, chacun, dans les cercles du pouvoir, devant feindre de croire à ce qu'il sait n'être qu'une fiction sur laquelle cependant reposent sa propre position, sa carrière et la réussite de ses ambitions.

De semblables résultats sont recherchés avec les apocryphes religieux du Japon des $\mathrm{XIX}^{\mathrm{e}}$ et $\mathrm{xx}^{\mathrm{e}}$ siècles (Jean-Pierre Berthon). Les fondateurs de nouvelles religions s'efforcent de créer de nouvelles communautés fondées sur la croyance en des mythes fondateurs originaux qu'ils inventent sans doute eux-mêmes mais en leur donnant une forme de sincérité en traçant des généalogies fictives aux récits faits. Prétendument conservés et transmis au sein de familles prestigieuses et anciennes, elles offrent un contrepoint saisissant à l'idéologie impériale et sont utilisés par les fondateurs de nouvelles religions qui sont aussi leurs auteurs. Leur exposition apparaît comme la mise au jour, l'invention, de vérités demeurées jusque-là cachées mais qu'il est devenu nécessaire de rendre publiques dans un processus de révélation au sens propre. Ces récits sont également des instruments dans la lutte politique, dans la mesure où ils touchent, spécialement dans les années 1920-1940, aux fondements mêmes de l'ordre idéologique sur lequel repose le Japon de la seconde guerre mondiale. Ils expriment ou font se cristalliser des ensembles d'idées qui permettent à certains groupes sociaux ou politiques de fonder des programmes sur un discours. C'est pour cette raison qu'ils sont, dans l'avant-guerre, violemment réprimés, les autorités constituées ne se trompant pas sur leur signification et leur finalité.

On ne saurait cependant qualifier de tels discours de mensongers. Les apocryphes écrits alors ont pour fonction de créer des communautés de croyance : le vrai, dès lors, n'est pas ce qui est vérifié scientifiquement, ce qui est établi par des preuves concrètes et irréfutables, mais ce qui est cru par tous comme étant tel. On entre ici dans le domaine de l'histoire des procédures par lesquelles des institutions ou des hommes parviennent à établir, enseigner, diffuser et reproduire un corpus de croyances : comment faire croire, c'est-à-dire assurer la conception, la diffusion ainsi que la réception d'un message religieux. Le recours à une histoire mythique, c'est-à-dire la fabrication de mythes d'origine est, en Occident, devenu impensable. Rappelons-nous toutefois que, durant le Moyen Âge, la culture savante élabora, pour expliquer le succès des Francs et leur rôle éminent dans l'histoire, le mythe de leur origine troyenne. Alors que les Romains descendaient d'Énée, les Francs, pour leur part, descendaient de Pharamond : la France de ce fait se trouvait justifiée à recueillir l'héritage de Rome, avec toutes les conséquences que l'on peut imaginer tant au point de vue idéologique que politique d'une telle position. Les mots ne sont pas les seuls vecteurs de l'idéologie. La monnaie est, elle aussi, normalement mobilisée pour cela. Elle est le document authentique par excellence puisque la frappe en Occident, la fonte en Chine, sont des monopoles d'État. Or, des perturbations parfois importantes peuvent se produire, qui font de ce document authentique une source d'erreurs historiques. C'est le propos de François Thierry analysant les systèmes de datation des monnaies chinoises. Des dysfonctionnements mineurs dans l'appareil d'État, par exemple des contre-ordres lancés à propos de la définition d'une monnaie, peuvent entraîner des conséquences en chaîne et faire apparaitre dans les séries monétaires soit des dates soit des événements qui n'ont pas 
existé. La complexité du système de datation chinois permet, voire favorise de telles erreurs qui sont le signe d'une sophistication et d'un raffinement extrême de l'appareil d'État, non celui d'une intention délictueuse. Celle-ci peut exister toutefois. Il peut être économiquement ou fiscalement nécessaire de mettre en circulation des monnaies d'une qualité moindre que celle courant habituellement dans le public. L'utilisation de types et de dates antérieurs est l'un des moyens possibles. C'est la technique normale utilisée au Moyen Âge en Occident pour mettre en circulation des monnaies dépréciées : les pièces ne portant aucune indication de valeur faciale, il suffit à l'autorité émettrice de frapper des pièces identiques à celles frappées précédemment mais contenant moins de métal fin ou pesant moins qu'elles ne le devraient. Ce moyen, utilisé par Philippe IV le Bel, fut à l'origine de la détestable réputation de faux-monnayeur de ce souverain qui s'essayait simplement aux manipulations monétaires dans un contexte économique tendu et troublé, sans avoir tout à fait les moyens de maîtriser les conséquences de ce qu'il faisait. Une particularité chinoise tout à fait remarquable est la fonte à l'étranger, aux frontières de l'Empire, de monnaies meilleures que celles circulant alors en Chine, utilisant les types chinois mais avec un module différent des originaux. Les souverains vietnamiens cherchaient à doter les marchands d'un moyen de paiement reconnu, facile à accepter du fait de la caution apportée (involontairement) par leur puissant voisin. Les Japonais, qui faisaient la même chose au XVI ${ }^{\mathrm{e}}$ siècle, pouvaient de la sorte solder plus aisément leurs achats en Asie du Sud-Est, leurs imitations étant de meilleure qualité que les monnaies circulant alors et, partant, davantage recherchées. En Occident, au même moment, on n'observe pas de phénomènes de même nature. Ils obligent en effet à un renoncement : la monnaie est normalement un signe de souveraineté des États qui, par elle, revendiquent à la fois leur autorité sur un territoire et leur prestige international. Lorsque l'on observe des mouvements de cette nature, il s'agit toujours de faux-monnayage, des États laissant s'implanter sur leur territoire des ateliers de faussaires qui nourrissent des circuits de distribution. Il s'agit normalement de billons, c'est-à-dire de monnaies à très faible pouvoir libératoire dont le manque se fait cruellement sentir dans les transactions marchandes à la fin du Moyen Âge. Le mouvement est donc inverse et la mise en circulation de telles espèces peut, dans certains cas limites, faire partie des instruments de lutte dans les conflits opposant les États entre eux.

Les manipulations monétaires permettant de falsifier des séquences historiques, en attribuant une frappe à un souverain qui n'est pas au pouvoir au moment où la fonte a réellement lieu, par exemple, quoique théoriquement possibles en Occident, n'y sont pas attestées. Ces points toutefois mériteraient d'être creusés : ils permettraient d'évaluer mieux encore que nous ne le faisons la capacité des États à contrôler effectivement la portée de leurs actes en matière monétaire. Les souverains savent-ils bien ce qu'ils font et sont-ils en mesure d'utiliser la monnaie comme instrument du discours historique, ce que font les Chinois? Jusqu'à présent, rien ne permet de formuler des hypothèses de cette nature pour l'Occident médiéval. Il vaudrait peut-être la peine d'y regarder de plus près, mais il ne semble pas probable que l'on ait des situations aussi clairement documentées que celles constatées en Chine où, en plus, les conditions juridiques et matérielles de l'émission monétaire ne sont pas du tout les mêmes qu'en Europe occidentale : le fait que l'État n'ait pas toujours revendiqué pour lui le monopole d'émission et que, de ce fait, des pratiques qui, en France passeraient pour du faux-monnayage y soient parfaitement licites, est forcément quelque peu déconcertant... 
17 La fraude sur les monnaies se trouve à l'intersection des préoccupations de cette série de textes : elle ressortit à la fois des altérations que l'on fait subir au statut des objets, soit en les plaçant dans un contexte qui n'est pas le leur, soit en les fabriquant autrement que comme ils devraient l'être et des modifications que l'on fait subir à la réalité historique en la rapportant de façon biaisée ou volontairement modifiée.

\section{Déformer la réalité} différemment, le mensonge pouvant être une nécessité sociale afin de créer des fictions plausibles auxquelles il est plus pratique de croire que de refuser de le faire. Dans le cas de la virginité des jeunes filles, la reconstitution de l'hymen perdu apparaît au Viêt Nam, comme l'un de ces mensonges socialement utiles voire nécessaires (Đinh Trọng Hiếu). La culture vietnamienne n'a pas avec les notions d'authenticité et de vérité les mêmes rapports que la culture européenne : dans une certaine mesure, la copie y vaut l'original et remplacer, dans un musée, un objet abîmé par un autre, neuf mais lui ressemblant, ne pose pas de problème particulier. Cette attitude étonnante pour un Européen peut avoir des prolongements en matière de présentation de soi et de mise en valeur de son propre corps : le recours aux postiches ou aux substituts ne faisant pas l'objet d'un jugement de valeur, l'argument de la mode et de la séduction l'emporte sur les autres considérations. Il $\mathrm{y}$ a là un terrain de fond sur lequel vient se greffer la question de la virginité des jeunes filles. Alors qu'elle est toujours valorisée à l'extrême, les transformations de la vie sociale et l'évolution des rapports entre les jeunes gens font que sa perte est fréquente, alors que la réprobation continue de s'abattre sur les jeunes filles qui se sont laissé séduire, spécialement dans le cas où elles n'ont pas épousé l'homme qui les a déflorées. L'hyménoplastie apparaît alors comme un moyen de réintégrer la norme sociale au prix d'une opération qui crée une illusion et établit la fiction de la « pureté » de la jeune fille au moment du mariage.

19 En France au Moyen Âge, les femmes sont vectrices de l'honneur familial. Jeune filles, elles doivent rester vierges, femmes, elles doivent être chastes. Une très forte réprobation s'abat sur les femmes ne se conformant pas au canon social, entraînant drames familiaux et déclassements. Il arrive en effet que les jeunes filles déflorées - le plus souvent il s'agit d'affaires de viol - soient chassées par leur père et n'aient plus aucun refuge dans la société ordinaire qui était la leur. Pour celles-là, aucune possibilité de réparation n'existait : dans ces conditions, la question de la tromperie que constitue l'hyménoplastie est une réponse adéquate à une pression devenue insoutenable du fait de la contradiction entre l'évolution des mœurs et les exigences d'une société demeurée sexuellement conservatrice.

20 Modifier son propre corps demeure toutefois l'exception. Les falsifications les plus fréquentes, les plus ordinaires, portent sur le capital immatériel des familles et en particulier, de façon presque spontanée, sur leur mémoire généalogique. Celle-ci conditionne en effet leur inscription dans un espace social lui-même ancré dans l'histoire: c'est le thème qu'aborde Guillaume Carré. Jusqu'à une date relativement récente, le nom de famille est demeuré au Japon le privilège des familles de la noblesse. Ce marqueur fort d'identité sociale et familiale était aussi un signe de distinction, une façon de se séparer des autres. Le nom pouvait aussi devenir un instrument de domination sociale : en autorisant leurs vassaux à utiliser leur nom, les grands lignages 
leur faisaient certes un cadeau inestimable grâce auquel ils étaient incorporés à une famille par une sorte d'adoption. Ils les empêchaient aussi d'acquérir toute forme d'autonomie et, surtout, d'identité, en dehors du groupe qui les dominait et les commandait. À un autre niveau, le choix d'un nom impliquant le choix d'un lignage d'appartenance, il orientait ou pouvait orienter les carrières auliques. Les membres de familles influentes prenaient donc la liberté de modifier leur nom et, avec lui, le passé tout entier de la famille. Choisissant un nom, ils revendiquaient avec lui le passé d'un groupe tout entier auquel ils étaient biologiquement étrangers. Ce fait pouvait être une prise de position politique ou un programme: affirmer appartenir à un groupe particulier, c'est aussi assumer son action, la mémoire s'attachant à son nom et affirmer que l'action qui sera désormais menée par les membres du groupe portant ce nom sera conforme à celle du passé.

21 Les ancêtres, dans le cas japonais, peuvent être choisis et abandonnés en fonction des besoins nés de circonstances politiques neuves. Il y a là une différence essentielle avec ce que nous comprenons de la situation occidentale. Au Moyen Âge, deux grands types de possibilité apparaissent. Ils se fondent dans l'ordre biologique mais produisent des effets qui sont des constructions de droit, des constructions sociales. La première possibilité est de privilégier la maison, organisme comportant des biens à la survie et à la transmission desquels les êtres humains sont voués. Tout est organisé pour que le patrimoine ne soit jamais divisé et qu'il soit transmis, de préférence en ligne masculine, mais ce n'est pas une nécessité absolue. La survie de la maison passant avant la transmission des caractères biologiques, il n'est pas anormal qu'un homme y entre par adoption, à condition qu'il épouse l'héritière et prenne son nom. Ici, aucune latitude d'action n'est laissée aux individus qui s'effacent devant l'impératif catégorique de la transmission du bien et du nom qui l'accompagne.

Dans les sociétés lignagères, au contraire, c'est le sang qui prime, et avec lui les caractères biologiques particuliers censés être portés par une famille et, plus particulièrement, par ses mâles. La transmission des biens est alors organisée de telle sorte que le groupe familial ait toutes les chances de survivre et de se développer : c'est ce qui se produit dans l'aristocratie où l'aînesse, avec son cortège de restrictions, n'apparaît que tardivement en France et ne se répand pas partout. Là aussi, pas de choix, mais un impératif, faire vivre un être moral, la famille, qui transcende chaque individu.

Ces choix ne sont pas universels. La difficulté avec laquelle le nom de famille s'impose, au $\mathrm{XIII}^{\mathrm{e}}$ siècle, et se généralise lentement, montre que la conscience d'appartenir à un groupe identitaire plus vaste que la cellule conjugale n'est pas universelle et se construit lentement au cours du temps. Si les familles bourgeoises de l'époque Edo se choisissent elles-mêmes un passé en désignant leur ancêtre et en construisant autour de lui une histoire familiale plus ou moins fictive reflétant en tout cas les partis pris par le ou les narrateurs, il n'en va pas de même des bourgeois français ou italiens qui, prenant un nom ou assumant un sobriquet dont on les affuble, ne vont pas jusqu'à construire des généalogies ou à revendiquer un passé familial. La construction de généalogies est une activité purement et exclusivement noble, née des nécessités politiques et sociales apparues au XII ${ }^{\mathrm{e}}$ siècle dans les milieux aristocratiques qui devaient consolider leurs positions face à l'accroissement de la puissance et du prestige des rois. Dans cette optique, tout en privilégiant la recherche et la mise en avant de la vérité sur la famille, c'est-à-dire en mettant en listes et en arbres généalogiques les ancêtres, un certain nombre de choix peuvent s'opérer: un homme, une femme ou une branche tout entière peuvent être 
oubliés alors que d'autres sont favorisés. Les listes d'ancêtres mentionnés dans les actes de fondation peuvent, dans ces conditions, être spectaculairement éclairantes, les relégations des moins illustrées ou des moins recommandables disant les choix opérés par la famille. Ces choix ne sont d'ailleurs pas irréversibles, les ancêtres oubliés pouvant être réintégrés.

Les familles bourgeoises, pour leur part, se définissent longtemps horizontalement, comme des groupes d'hommes et de femmes contemporains se connaissant et dont les relations ne sont pas surdéterminées par le fait de descendre d'un même ancêtre. Les obligations, et en particulier, les obligations d'entraide, sont fort limitées à l'intérieur de ce groupe et naissent autant de l'amitié et de la convergence des intérêts que des devoirs que l'appartenance à une même descendance pourrait faire naître. Dans le monde urbain, le désir d'ancrer le groupe familial dans le temps n'apparaît qu'à la fin du Moyen Âge avec les livres de raison dont la justification est tout autant économique que sociale ou familiale. La mémoire familiale reflète alors non des sélections et des choix mais la réalité des faits, à la manière de l'état-civil, en quelque sorte.

Pour un médiéviste, la ductilité des structures familiales japonaises est ainsi une source d'étonnement, en même temps qu'un appel à approfondir ses propres réflexions sur la nature même de la famille médiévale en Occident.

Enfin, un dernier point est abordé par Christian Lamouroux et Guillaume Carré, celui des contrefaçons de marchandises et de la falsification des produits dans la Chine et le Japon prémodernes. Le texte est un pari : il compare la Chine des $\mathrm{x}^{\mathrm{e}}$-XIII ${ }^{\mathrm{e}}$ siècles au Japon des $\mathrm{XVII}^{\mathrm{e}}-\mathrm{XIX}{ }^{\mathrm{e}}$ siècles, proposant l'idée que situations sociales, structures et dynamiques économiques y sont analogues et que les deux États y affrontent, à l'égard de la régularité des transactions, des problèmes similaires les obligeant à proposer des solutions propres à chacun, mais parfaitement comparables. Japonais et Chinois sont amenés en effet à se montrer souples sur la qualité et l'authenticité, tout en semblant prendre des mesures pour garantir l'une et l'autre. Ils sont en effet pris dans une contradiction tout à fait classique dans laquelle le bon approvisionnement des marchés et l'ordre social ne font pas nécessairement bon ménage avec une absolue recherche de la qualité. La volonté de contraindre les producteurs à travailler de façon loyale et à produire des objets dont les qualités puissent être vérifiées sans difficulté se heurte à la nécessité de satisfaire rapidement aux besoins des consommateurs. Entre d'autre part ici une composante éthique : les marchands comme les fabricants, quels que puissent être les discours des gouvernants sur la nécessité de produire de bonnes marchandises et de les échanger au juste prix, sont parfois assez peu regardants sur la qualité et exigeants sur le prix, quelles que puissent être, par ailleurs, les proclamations des métiers et la dureté de réglementations difficiles à appliquer dans le détail.

En Occident, on admet encore peut-être trop facilement, du fait d'une documentation essentiellement normative, que les règlements étaient effectivement appliqués et que les métiers assuraient la victoire d'une idéologie de la qualité au détriment de la satisfaction des besoins locaux. Il est de fait que la première industrialisation de l'Occident s'est fondée à la fois sur les échanges à longue distance et la fabrication de produits susceptibles de justifier de longs transports et les coûts afférents à ceux-ci. Or, il est patent que, dès le XIII ${ }^{e}$ siècle, la population urbaine atteint des niveaux tels que les questions d'approvisionnement des marchés en biens de consommation de qualité médiocre, voire carrément basse, sont devenues importantes sinon vitales. Elles se posent peut-être dans les mêmes termes que dans la Chine des Song ou le Japon des XVII ${ }^{\mathrm{e}}$-XIX ${ }^{\mathrm{e}}$ 
siècles où il faut en rabattre sur la qualité comme sur l'intensité des contrôles pour pouvoir assurer une alimentation pérenne des marchés. En même temps cependant, la question du rapport au produit authentique demeure. Elle est prônée en Occident comme une valeur en soi par les métiers et, en Asie orientale, est protégée par les autorités. Il n'empêche que les tromperies sur la marchandise existent. Elles semblent être moins mal considérées en Asie orientale qu'en Occident à conditions sociales et politiques similaires. Là aussi, sous bénéfice d'inventaire: il faudrait y voir de plus près, analyser les procédures de contrôle et s'interroger sur leur réelle efficience en Occident ainsi que sur la valeur effectivement attribuée au produit authentique par les agents économiques de la période $\mathrm{XII}^{\mathrm{e}}-\mathrm{XV}^{\mathrm{e}}$ siècles.

Il est un secteur toutefois où l'Occident a agi de façon très différente de ce que l'on voit en Asie orientale, c'est celui des prix. Alors que les Song pratiquent une politique de prix administrés ou tentent de le faire, jamais aucune monarchie médiévale n'a eu les moyens matériels, ni les connaissances économiques suffisantes pour proposer des solutions de cette nature. Le système de surveillance des marchés ne touchait que les monnaies, les poids et mesures et, évidemment, la police des marchés. Le souci d'ordre sur ces derniers était d'ailleurs l'une des motivations les plus puissantes d'intervention, avec la volonté de peser sur les prix afin de faire en sorte que les salaires urbains demeurent à un bas niveau. Aucun État, sauf peut-être les villes italiennes à partir du XII ${ }^{\mathrm{e}}$ siècle, n'a disposé en Occident des mêmes capacités de contrôle et d'intervention que celles détenues par les autorités chinoises ou japonaises.

Enfin, la question de l'évolution des techniques et des savoir-faire a amené à poser de façon nouvelle la question du faux et de l'écart du produit fini par rapport à la norme définie par une instance quelle qu'elle soit. Ce sont là des faits désormais bien connus pour la France de la fin du Moyen Âge, s'agissant de produits incorporant beaucoup de savoir-faire comme les fers et les aciers du sud-ouest du royaume. Là, il ne peut y avoir de qualité unique, bien définie, mais des références ponctuelles prises parmi des productions bien individualisées. Les produits de telle ou telle forge ont, dans la seconde moitié du $\mathrm{xv}^{\mathrm{e}}$ siècle, une réputation établie d'après la qualité du minerai utilisé et d'après la connaissance du savoir-faire du propriétaire de l'installation. C'est par rapport à ces indications que les autres métaux sont appréciés sur le marché et qu'une valeur peut leur être attribuée. Il n'est plus question ici de falsification mais de réputation et de connaissance des produits par les hommes achetant et vendant sur les marchés. Il ne s'agit ici que d'un petit milieu fonctionnant presque en interconnaissance, à la jonction des mondes urbains et ruraux. L'État ici ne peut intervenir efficacement, pas plus d'ailleurs que les autorités urbaines ou seigneuriales, et c'est une forme d'auto-contrôle des agents qui assure la stabilité des échanges et le bon fonctionnement des marchés.

L'État, enfin, intervient comme acteur sur les marchés orientaux, parce qu'il est gros consommateur, ce qu'il n'est pas en Occident, en tant que tel, du moins avant la seconde moitié du XIV e siècle, avec le maintien d'un état de guerre permanent et l'apparition d'une fiscalité royale. Il y a là une différence substantielle entre les sociétés orientale et occidentale, à niveau de développement égal. L'État, en Occident n'intervient finalement que pour contrôler le bon fonctionnement d'organismes, de métiers, qui se sont développés en dehors de lui et sans qu'il puisse intervenir ou cherche même à le faire.

31 L'ampleur des problèmes soulevés par les auteurs du volume nous renvoie sans arrêt à l'état de la réflexion historique sur les sociétés occidentales. Médiéviste, j'ai naturellement privilégié les questions d'histoire du Moyen Âge. Les questions d'ordre très 
général, le faux, la falsification, mais aussi, par conséquent, le contrôle et la répression, sont également présentes à tous les niveaux dans les réflexions des historiens occidentalistes. L'intérêt d'un volume comme celui-ci est de leur donner à penser en proposant des situations analogues mais certainement pas identiques à celles qu'ils connaissent en Occident, tout en leur rappelant que la pluralité des civilisations n'empêche pas une position commune des problèmes.

\section{RÉSUMÉS}

Le faux et la falsification atteignent, en Orient comme dans le reste du monde, l'ensemble des compartiments de la vie sociale. Ils posent des problèmes d'ordre éthique, ou de morale pratique, s'insèrent dans le débat idéologique ainsi que dans les pratiques familiales ou la vie économique. Les textes proposés dans ce volume et que ce papier commente, présentent, à travers des exemples et des situations assez divers dans le temps et concernant un espace très vaste, une gamme assez large des problèmes que pose la question du faux dans la vie sociale. La falsification est dans certains cas - hyménoplastie, reconstruction de généalogies - une manipulation effectuée avec des objectifs restreints pour des propos privés, relevant de la réussite sociale, individuelle et collective. Il s'agit de tricheries limitées visant à assurer la conformité d'une personne aux règles du jeu social. Dans d'autres cas - archéologie paléolithique, abdications impériales -, ces manipulations sont opérées non pour obtenir ce résultat, mais pour modifier le jeu lui-même. Enfin, au cœur de la vie économique, falsifications et faux posent la question du contrôle des institutions de l'état sur la production et les échanges, à la fois pour des raisons ayant trait à l'ordre social et à sa stabilité et, de façon très prosaïque aussi, pour des motifs fiscaux. La qualité des produits fabriqués, et donc la possibilité de les identifier de façon sûre, est également un point vital pour toute économie ayant cessé d'être une pure économie de subsistance et reposant autant sur l'échange que sur la production.

Fakery and falsification, in the East as well as in the rest of the world, appear in all realms of social life, raising issues of ethics and practical morals that arise in ideological debates as well as in family practices and economic life. The articles published in this volume and discussed in the present paper present various examples, diverse in time and covering a vast space, of a fairly wide range of problems posed by the issue of fakes in social life. In some cases the falsification concerns a private matter - hymenoplasty, the reconstitution of a genealogy - and is a manipulation associated with private objectives of social success, individual or collective. Such deceits are limited and aimed at ensuring that a person conforms to the rules of social life. In other cases - Paleolithic archaeology, imperial abdication - the aim of the manipulation is to modify the rules themselves. Finally, at the very heart of economic life, falsification and fakes raise the question of State institutional control over manufacture and business transactions, for reasons related social order and social stability and, more prosaically, for fiscal reasons. The quality of manufactured goods, and therefore the possibility of evaluating them, is vital in any economy that has left the purely subsistence stage to rely on production and exchange. 


\section{AUTEUR}

\section{LAURENT FELLER}

Laurent Feller,ancien membre de l'École Française de Rome, est professeur d'histoire sociale de l'Occident médiéval à l'Université Paris I. Il a publié : « Les mouvements migratoires en Italie durant le haut Moyen Âge : déplacements contraints, négociés ou spontanés ? ", XxxIV semana de Estudios Medievales, Estella, 16-20 de julio 2007, Espagne (2007) ; « Dette, stratégies matrimoniales et institution d'héritier : sur l'élite paysanne lombarde au IX ${ }^{\mathrm{e}}$ siècle », Revue Historique, 645/2 (2008), p. 339-368. URL: http://www.univ-paris1.fr/recherche/page-perso/page/? page=publications\&uid=feller 